\title{
Title: Causal illusion as a cognitive basis of pseudoscientific beliefs
}

\author{
Short title: Causal illusions and pseudoscience \\ Marta N. Torres ${ }^{1,2}$, Itxaso Barberia ${ }^{1}$, Javier Rodríguez-Ferreiro ${ }^{1,2,3^{*}}$ \\ 1 Departament de Cognició, Desenvolupament y Psicologia de la Educació, Universitat \\ de Barcelona, Barcelona, Spain \\ 2 Institut de Neurociències, Universitat de Barcelona, Barcelona, Spain \\ ${ }^{3}$ Grup de Recerca en Cognició i Llenguatge, Universitat de Barcelona, Barcelona, \\ Spain
}

${ }^{*}$ Corresponding author information: Javier Rodríguez Ferreiro, Passeig de la Vall d'Hebron, 171,

08035 Barcelona, Spain, (e-mail: rodriguezferreiro@ub.edu).

\begin{abstract}
:
Causal illusion has been proposed as a cognitive mediator of pseudoscientific beliefs. However, previous studies have only tested the association between this cognitive bias and a closely related but different type of unwarranted beliefs, those related to superstition and paranormal phenomena. Participants $(n=225)$ responded to a novel questionnaire of pseudoscientific beliefs designed for this study. They also completed a contingency learning task in which a possible cause, infusion intake, and a desired effect, headache remission, were actually noncontingent. Volunteers with higher scores on the questionnaire also presented stronger causal illusion effects. These results support the hypothesis that causal illusions might play a fundamental role in the endorsement of pseudoscientific beliefs.
\end{abstract}

\section{Keywords:}

causal illusion; pseudoscience; paranormal beliefs; superstition; cognitive bias; illusion of causality

\section{Data availability statement:}

The data that support the findings of this study are provided as supporting information.

\section{Acknowledgements:}

This study was supported by the grants PSI2016-75776-R (AEI/FEDER, UE) to IB and PSI2016-80061-R, (AEI/FEDER, UE) to JRF, both from the Agencia Estatal de Investigación of the Spanish government and the European Regional Development Fund, as well as grant 2017SGR387 (AGAUR) from the Catalan government to JRF. 
Previous studies have aimed to identify the mechanisms underlying unwarranted beliefs related to paranormal phenomena (Blackmore \& Trościanko, 1985; Brugger, Landis, \& Regard, 1990; van Prooijen, Douglas, \& De Inocencio, 2018; Wiseman \& Watt, 2006). In this study we focus on a different, though closely related, domain of unwarranted beliefs: those related to pseudoscience. According to the demarcation criteria adopted by Fasce and Picó (2019, p. 618), for something to be considered a pseudoscience, it needs to be "presented as scientific knowledge" (A), and also meet at least one of the following three conditions: "refers to entities and/or processes outside the domain of science" (B), and/or "makes use of a deficient methodology" (C), and/or "is not supported by evidence" (D). As noted by these authors, the difference between pseudoscientific and paranormal beliefs lies in the fact that although the latter still refers to aspects outside the domain of science (it fulfills B), it is not presented as scientific knowledge (does not fulfill A). While paranormal and pseudoscientific beliefs tend to positively correlate (Fasce \& Picó, 2019; Lindeman, 2011; Majima, 2015), they present different prevalence rates in the population. For instance, according to a national survey on social perception of science conducted in Spain (FECYT, 2017), whereas only $22.4 \%$ and $27.5 \%$ of the population respectively believe in paranormal phenomena and superstitions (i.e. lucky charms or numbers), when asked regarding the effectiveness of pseudoscientific treatments, the percentages rise to $52.7 \%$ for homeopathy and $59.9 \%$ for acupuncture.

Understanding the cognitive mechanisms supporting pseudoscientific beliefs is especially relevant because, unlike what happens in relation to paranormal beliefs, which are negatively related to education level (Aarnio \& Lindeman, 2005), they have been shown to be more present in individuals with higher education levels (NCCIH-NIH, 2008), and endorsement of these kinds of claims is noteworthy even among educated professionals such as physicians (Posadzki, Alotaibi, \& Ernst, 2012) or teachers (Ferrero, Garaizar, \& Vadillo, 2016).

Given that much putative pseudoscience (for instance, that related to medical treatments) relies on causal relations, it has been proposed that causal illusions might be a fundamental cognitive 
basis of pseudoscientific beliefs (Matute, Yarritu, \& Vadillo, 2011). The terms causal illusion or illusion of causality refer to a cognitive bias leading one to perceive a causal connection between two events which are actually non-contingent (Matute et al., 2015).

To our knowledge, there have been two recent attempts to explore the relationship between the scores obtained in questionnaires measuring unwarranted beliefs and the intensity of the causal illusions generated in null contingency learning tasks. First, Blanco, Barberia and Matute (2015) found that individual differences in the number of paranormal beliefs held by a group of participants, as measured by the Revised Paranormal Beliefs Scale (RPBS, Tobacyk, 2004) in its Spanish version (RPBS-Sp, Díaz-Vilela \& Álvarez, 2004), predicted differential propensity to develop causal illusions. Blanco et al. (2015) presented their participants with the records of several fictitious patients who allegedly suffered from the same disease. The volunteers could then decide whether to administer each patient a given drug or not. Immediately afterwards, they were told whether the patient healed or not. Note that, in this task, the two binary variables for which the contingency is being assessed are conceived as events (taking the drug; recovering from the disease) vs. non-events (no drug; no recovery), and, therefore, it can be considered an asymmetric contingency learning task (Allan, 1993). After the volunteers had gone through all the patients, they evaluated the effectiveness of the drug on a numerical scale from 0 (the drug was ineffective) to 100 (the drug was perfectly effective). The chances of recovery were set at $75 \%$ irrespective of the administration of the drug and, therefore, the drug did not increase the probability that a patient would show recovery (here we are focusing on their noncontingent condition, but the authors also included a contingent condition in their design). Given this null contingency between drug administration and recovery, higher ratings in the numerical effectiveness scale were treated as indicative of a greater causal illusion developed by the participants. In Blanco et al.'s results, the relation between paranormal beliefs and causal illusion was mediated by the proportion of patients to which each participant decided to administer the drug, leading the authors to conclude that the way in which individuals expose 
themselves to available information might play a crucial role in the relation between paranormal beliefs and causal illusion.

The other noteworthy work regarding the relationship between causal illusions and misbeliefs is a recent study by Griffiths, Shehabi, Murphy and Le Pelley (2018). In their study, Griffiths et al. (2018) asked their participants to discover the extent to which pressing a button controlled the illumination of a light. Again, the task involved a null contingency between the button press and the illumination of the light, i.e., the light illuminated about $60 \%$ of the time independent of the participant pressing or not pressing the button. Similar to the procedure used by Blanco et al., after completing the task the participants were asked to evaluate the extent to which the action of button-pressing controlled the illumination of the light, on a numerical scale ranging from 0 (meaning no control) to 100 (meaning total control). These authors showed that differential scores in superstitious beliefs, as measured by the Superstitious Beliefs Questionnaire (SBQ, developed ad hoc for their study), were positively correlated with the intensity of the causal illusions developed in their contingency learning task. Griffiths et al. instructed their participants to press the button on about half the occasions and not to press it on the other half. Interestingly, even when controlling for spontaneous individual differences in the behavioral component by instructing participants about how to behave during the task, these authors still found a positive correlation between the intensity of the causal illusions developed by the participants and their scores on the SBQ. Griffiths et al. concluded that their findings were complementary to those of Blanco et al. (2015) by showing that the differences between superstitious and non-superstitious individuals relied on the way they interpreted the experienced cause-effect contingencies.

Note that the questionnaires employed by each of the preceding studies differed. Blanco et al. (2015) opted for the RPBS-Sp, which includes items distributed across eight dimensions: witchcraft, psi, traditional religious beliefs, spiritualism, extraterrestrial life and actual visits, precognition, superstition and extraordinary life forms (Díaz-Vilela \& Álvarez, 2004). Griffiths 
et al. instead developed a new questionnaire because they argued that the RPBS and other measures "contained statements that have not yet or cannot be verified, but which may be rational beliefs ('There is life on other planets') or items that have little bearing on daily life ('The abominable snowman of Tibet exists')" (Griffiths et al., 2018, pp. 504-505). In its place, they developed items "to reflect beliefs held in the community, which address implausible causal relationships, and for which evidence (either for or against the belief) is likely to be encountered in ordinary life" (Griffiths et al., 2018, p. 505). When going through the items chosen by Griffiths et al. (2018, see their appendix B), we can find statements related to subscales already present in the RPBS-Sp, such as items related to superstition ("If I passed a ladder I would walk around it rather than underneath it") or religion ["I believe in the existence of a higher being (such as a Christian God, Allah, Shiva, Waheguru, or Satan)"], but also items related to pseudoscientific disciplines such as homeopathy ['"Alternative' therapies (such as homeopathic remedies, aromatherapy, reflexology, chiropractic manipulation, or therapy based on the body's energy fields) can be an effective way of treating illnesses and ailments"] or graphology ("It is possible to gain information about a person's personality by analysing their handwriting").

Along with the theoretical basis outlined above, the fact that Griffiths et al. (2018) observed a significant correlation between the intensity of causal illusions and scores on a questionnaire partially consisting of items related to pseudoscientific beliefs, inspired us to assess the specific relation between causal illusions developed in the laboratory and misbeliefs specifically related to pseudoscience. To do this, we measured our participants' pseudoscientific beliefs and presented them with a contingency learning task. In order to mirror a situation specifically related to pseudoscience, the contingency learning task used a pseudomedicine-related scenario in which participants were asked to decide whether a given infusion was effective in reducing headache. In contrast to the procedure used by Blanco et al. (2015), in which volunteers decided whether or not to administer the drug to the patients, our participants were passively presented with the information regarding whether the patients used the infusion and whether they 
recovered from the headache. In this sense, our task is more similar to the one used by Griffiths et al. (2018), who controlled for the rate of cause administration by asking their participants to keep a constant rate of cause administration. However, instead of presenting participants with balanced samples of patients taking and not taking the infusion, we presented them with a majority of patients taking the infusion. Specifically, $75 \%$ of patients took the infusion whereas only $25 \%$ did not take it ( $75 \%$ of patients recovered irrespective of the intake of the infusion, see Barberia, Vadillo, \& Rodríguez-Ferreiro, 2019 for another study using the same frequencies). These frequencies were used in order to maximize the causal illusion effect in our participants, as previous studies have shown that passive contingency learning tasks in which the potential cause is frequently present yield stronger illusion effects, especially when the outcome also occurs with a high frequency (e.g. Blanco, Matute, \& Vadillo, 2013). Taking this into account, our hypothesis is that individuals with higher scores in a scale measuring pseudoscientific beliefs will rate the infusion as more effective, thus displaying stronger causal illusions than those with lower scores. Given the passive nature of our task, this result would be indicative of a bias in the interpretation of available contingency information. Finally, in order to replicate the results obtained in previous studies, we also included a measure of superstitious beliefs (Griffiths et al., 2018) in our experimental design.

\section{Method}

\section{Participants}

225 psychology students from the University of Barcelona (44 males and 181 females) participated in this study. Ages ranged from 20 to 64, with a mean of 22.79 years old $(\mathrm{SD}=$ 6.05). The study protocols were approved by the ethics committee of the university (Institutional Review Board IRB00003099, Comissió de Bioètica de la Universitat de Barcelona). Participants provided informed consent before their participation. 


\section{Materials}

\section{Contingency task}

The task was an adaptation of the standard task employed in the literature on causal illusions (e.g., Blanco et al., 2013). Participants were asked to judge the ability of an infusion of an herb brought from the Amazon to heal headache (the Amazônia task). The participants viewed, on a computer screen, a series of medical records (one per trial) describing patients suffering a headache. In each trial, the participants were shown whether a given patient received the infusion or not, and they were asked (yes/no question) if they thought the patient would heal in the subsequent two hours. Then, the participants received feedback indicating whether the patient was healed. After observing all patients, the volunteers were presented with an effectiveness question (i.e. "To what extent do you think the herb infusion is effective as a cure for headache? Provide a number between 0 and 100 where 0 means not effective at all and 100 means totally effective”; original question in Spanish: “¿Hasta qué punto crees que la infusión de hierbas es efectiva contra el dolor de cabeza? Introduce un número entre 0 y 100 . Un valor de 0 significa que no es nada efectiva y un valor de 100 que es totalmente efectiva" ). The infusion was completely ineffective, as healing rates were noncontingent on the administration of the infusion: $\mathrm{P}($ Healing $\mid$ Infusion $)=\mathrm{P}($ Healing $\mid \neg$ Infusion $)=0.75$, making the contingency 0 . Specifically, participants observed a total of 48 patients. The infusion was administered to 36 of them, from which the headache disappeared in 27 cases and persisted in 9 . The infusion was not administered to the remaining 12 patients, from which the headache disappeared in 9 cases and persisted in 3 . The different trial types were randomly presented to each participant.

For exploratory reasons, we also measured participants' recall of the frequencies of the four different trial types experienced during the task (e.g. "In how many patients who took the infusion did the headache disappear?"). About half of the participants responded to these questions before the effectiveness one. 


\section{Pseudoscience Endorsement Scale}

We designed the Pseudoscience Endorsement Scale (PES) to be used in our study. All instructions and items for the PES are listed in Appendix 1. The scale consists of 20 items referring to popular pseudoscientific myths (e.g. the preventive impact of a positive attitude over cancer, stress being the primary cause for ulcers, the use of polygraph as a lie detection mechanism..., see Lilienfeld, Lynn, Ruscio, \& Beyerstein, 2010) and disciplines (e.g. homeopathy, Reiki, Bach flowers, graphology, neuro-linguistic programming...). Three of the items (items 1, 15 and 20) were adapted from the Belief in the non-Paranormal Pseudoscience Scale by Majima (2015). Each item consisted of a statement that the participants had to rate on a scale from 1 (i.e., "Strongly disagree") to 7 (i.e., "Strongly agree"). Note that, according to Fasce and Picó (2019), a claim can be considered pseudoscience if it is presented as scientific knowledge but is not supported by evidence. Therefore, the pseudoscientific status of a myth or discipline is not necessarily immutable and can change in the light of new evidence. Moreover, the fact that a topic is considered pseudoscientific does not imply that studies investigating the topic are themselves pseudoscientific.

In order to verify the reliability of the PES, a total of 143 psychology students from the University of Barcelona (122 females, 19 males, and 2 participants who did not disclose their gender), different from those who took part in the experiment, completed this scale. Ages ranged from 21 to 54 , with a mean of 22.89 years old $(S D=3.89)$. A reliability analysis on the PES data (mean $=3.47, S D=0.83$ ) performed with IBM SPSS Statistics (version 23.0.0.2) showed high internal consistency of item scores, Cronbach's $\alpha=0.89$. Hotelling's $T^{2}$ index of equality was $T^{2}=897.28, F(19,124)=41.24, p<.001$; and Tukey's test of nonadditivity was $F(1,2698)=1.02, p=.312$. Thus, all the items were interrelated and additive. Then, we tested the suitability of our data for the Principal Components Analysis (PCA). The Kaiser-MeyerOlkin (KMO) test showed a high measure of sampling adequacy, $\mathrm{KMO}=0.87$, and Bartlett's test of sphericity was significant, $\chi^{2}(190)=930.21, p<.001$, showing high correlation between items. The PCA showed five components with eigenvalues over 1.0, which explained 32.48\%, 
$7.61 \%, 6.13 \%, 5.84 \%$, and $5.11 \%$ of the variance. According to the PCA, most items had a higher load in component 1 and the variance percentages explained by the other four components were very low. A parallel analysis was conducted, which extracted only one component. Given these results, it seems legitimate to accept the parallel analysis solution, based in only one component (general pseudoscientific beliefs).

A total of 141 of these participants also responded to the RPBS-Sp (Díaz-Vilela \& Álvarez, 2004). Following Barberia, Tubau, Matute, and Rodríguez-Ferreiro (2018), we slightly reworded item 20 and did not consider scores corresponding to item 23 to calculate the global score. Mean global scores for this scale were $1.92(S D=0.70)$. The Shapiro-Wilk test for normality showed that the mean scores on RPBS-Sp did not follow a normal distribution, $W(141)=0.92, p<.001$. Thus, we conducted the non-parametrical Kendall's tau test to analyse the correlation between scores on the RPBS-Sp and the PES, which returned a positive correlation, $r_{\tau}=0.34, p<.001$.

It should be noted that another interesting scale for measuring pseudoscientific beliefs has been published since we designed the PES and gathered these initial data for testing its reliability. This is the Pseudoscientific Beliefs Scale by Fasce and Picó (2019). However, we believe that our scale might be more adequate for our purposes here because it specifically focuses on pseudoscience, whereas that of Fasce and Picó (2019) includes both items related to pseudoscience as well as to science denialism, a close but conceptually different subcategory of unwarranted beliefs. In any case, Fasce and Picó's (2019) scale constitutes an alternative for measuring pseudoscientific beliefs that might also be employed in future studies on causal illusions.

\section{Superstitious Beliefs Questionnaire}

We translated the English SBQ (Griffiths et al., 2018) into Spanish following common translation and back-translation procedures (Sierro, Rossier, Mason, \& Mohr, 2016). Thus, a 
Spanish speaker of advanced English proficiency translated the English version into Spanish. Then, an English-native bilingual translator back-translated the Spanish version. The minor differences revealed by comparing the two versions were discussed by the two translators until agreement was reached. Our participants completed this Spanish version of the SBQ. The statements were rated by the participants on a scale from 0 (i.e., "Strongly disagree") to 4 (i.e., "Strongly agree").

\section{Procedure}

The participants completed first the Amazônia computerized contingency learning task followed by the PES, designed for the present study, and the SBQ (Griffiths et al., 2018), in that order.

\section{Results}

The dataset can be found at https://osf.io/w29cs/. Data were analysed with JASP (version 0.9.2.0). We performed Bayesian t-tests using JASP's default Cauchy prior width, $r=0.707$. We interpreted Bayes factors (BF) following Table 1 in Wagenmakers et al. (2018). Given that the Shapiro-Wilk test showed that the mean scores on the contingency task, $W(225)=0.87, p<.001$, and the mean scores on the SBQ, $W(225)=0.92, p<.001$, did not follow a normal distribution, we opted to conduct Kendall's tau for testing all correlations.

Regarding the contingency task, the mean of effectiveness judgements (i.e. casual illusion) was significantly higher than zero, mean $=63.22, S D=21.07, t(224)=45.00, p<.001, B F_{10}=$ $1.995 \mathrm{e}+110$. These results suggest that the participants perceived the task as contingent, developing, at least to some extent, a causal illusion.

Per-participant subjective probability contrasts [P(Healing|Infusion) - P(Healing| $\neg$ Infusion $)$, calculated from their responses to the exploratory frequency recall questions, significantly correlated with the causal illusion, $r=.543, p<.001, B F_{10}=3.653 \mathrm{e}+15$. This result suggests 
that the relative recall of different trial types by the participants could be, somehow, related to their perception of a causal relation between the infusion and the disappearance of the headache. Given that question presentation order did not affect the causal illusion $(F<1)$, we ignore these exploratory questions in the following.

Both the reliability of the PES and the SBQ were high for the experimental sample, $\alpha=0.91$ and $\alpha=0.93$, respectively. In general, scores on the PES, mean $=3.30$ (on a 1 to 7 scale), $S D=1.02$, appeared to be relatively higher than those corresponding to the SBQ, mean $=0.87$ (on a 0 to 4 scale), $S D=0.69$. Crucially, a Kendall correlation analysis between causal illusion scores on the contingency task and scores on the PES showed that they were positively correlated, $r_{\tau}=0.13, p$ $=.007, B F_{10}=4.76$ (see Figure 1, left panel). The PES and the SBQ were significantly correlated, $r_{\tau}=0.47, p<.001, B F_{10}=1.939 \mathrm{e}+22$. Nevertheless, in contrast to the results obtained by Griffiths et al. (2018), we observed no significant correlation between the causal judgements and the SBQ scores, $r_{\tau}=0.09, p=.068, B F_{10}=0.55$ (see Figure 1 , right panel). For the sake of comparison with previous studies (Blanco et al., 2015; Griffiths et al., 2018), we also performed Pearson correlations, which showed that scores on the PES were positively correlated with both causal illusion, $r=0.22, p<.001, B F_{10}=23.96$, and scores on the $\mathrm{SBQ}, r=0.63, p<$ $.001, B F_{10}=1.597 \mathrm{e}+23$. According to this analysis, the correlation between scores on the SBQ and the intensity of the causal illusion was, again, not significant, $r=0.11, p=.105, B F_{10}=$ 0.31 .

All in all, attending to the results of the previous Bayesian analyses, our data provide moderateto-strong evidence favouring the existence of a positive association between scores on the PES and causal illusion. In contrast, our results offer anecdotal-to-moderate evidence against the association between causal illusion and scores on the SBQ

Figure 1 about here 


\section{Discussion}

In this research, we aimed to assess the relation between causal illusion and belief in pseudoscience. Our data show that participants with higher scores on a novel scale specifically designed to measure pseudoscientific beliefs also developed stronger causal illusions in a contingency learning task with zero contingency. Although the rate of headache remission in the experimental task was independent of the patients taking or not taking the infusion, most of the participants perceived some degree of causal relation between infusion intake and healing. Crucially, volunteers with higher pseudoscientific beliefs rated the causal relation as stronger than those with low pseudoscientific beliefs.

Our study elaborated on previous research by Blanco et al. (2015) and Griffiths et al. (2018), who observed that volunteers with higher levels of, respectively, paranormal and superstitious beliefs also tended to develop stronger causal illusions during contingency learning tasks. We extended these findings to the field of pseudoscience, which is closely related to paranormal beliefs, but presents its own characteristics (Fasce \& Picó, 2019). Our data, nevertheless, did not replicate the association between superstitious beliefs, as measured by the SBQ, and causal illusion, as we failed to observe a significant correlation between these two variables. We consider that the lack of significant effects in this regard could have been due to a floor effect as evidenced by the extremely low scores obtained by our participants on the superstitious beliefs scale. In our view, this null effect, in fact, stresses the relevance of this study, indicating that, whereas superstition might not be that relevant in our context, pseudoscientific beliefs appear to be more widespread.

As noted by the reviewers of an early version of this article, an alternative explanation for the discrepancy between our results and those of Griffiths et al. (2018) is that our contingency learning task was framed in terms of a natural remedy, an herb from the Amazon, a scenario that might parallel that of pseudomedicines. In contrast, Griffiths et al. (2018), used a more neutral 
scenario referring to discovering the connection between pressing a button and the illumination of a light. In this sense, it is possible that those participants who endorsed more pseudoscientific beliefs were, from the start, more inclined to believe in the natural remedy, independent of the contingency information observed during our task. Note, besides, that differences in the cover story used could also be responsible for the lack of replication of the significant correlation between superstitious beliefs and causal illusion observed by these authors. In our view, employing contingency learning tasks with content-relevant cover stories might more accurately mimic the conditions in which those beliefs develop in real life. Nevertheless, future research should explore if the correlation we observed between causal illusions and pseudoscientific beliefs also appears when the contingency learning task refers to a more neutral scenario, such as that used by Griffiths et al. (2018).

Regarding the specific cognitive mechanisms supporting the observed effect, in the study by Blanco et al. (2015) the association between belief in the paranormal and causal illusion had been shown to be mediated by the volunteers' tendency to administer the drug to the patients, leading the authors to conclude that the individuals' information search strategies played a crucial role in their observed effect. In our study, the fact that participants were passively presented with the information (i.e. they could not manipulate the administration of the infusion during the task), indicates that the association between pseudoscientific beliefs and causal illusion also relies on the way individuals interpret given information. In this sense, our results are analogous to those obtained by Griffiths et al. (2018) although, in our case, the association is specifically drawn between causal illusion and pseudoscientific beliefs. From their perspective, the key aspect to understand the association between unwarranted beliefs and causal illusion could be a biased interpretation of the co-occurrences of events so that believers underestimate the likelihood of these coincidences (in our asymmetrical contingency learning task the events being having the infusion and recovering from the headache) and, hence, overestimate their relevance when taking them into account during the causal judgement. This hypothesis is rooted in previous evidence associating the random number generation bias (i.e. the tendency to avoid 
number repetition when trying to produce sequences of random numbers) to paranormal beliefs (Brugger et al., 1990). The general claim is that believers overestimate the relevance of coincidences because they misunderstand the probability of these coincidences occurring by chance due to an inaccurate representation of randomness.

Alternatively, it could be the case that believers generally present a stronger propensity to connect separate events (Bressan, 2002), in line with the results of studies showing that those who believe in the paranormal tend to perceive more meaningful patterns in random visual noise than non-believers (Brugger et al., 1993). From this perspective, individuals would vary in the amount of evidence they require to accept or reject a given hypothesis, with believers being more inclined to accept causal explanations for coincidences in general (Brugger \& Graves, 1997).

Although our results indicate the existence of a relevant relation between endorsement of pseudoscientific beliefs and a tendency to develop causal illusions, we emphasise that the correlational nature of our study does not allow the establishment of a causal relation between these two variables. Nor does it allow one to ascertain the direction of a putative causal relation. Through this paper, we outline the possibility that a cognitive bias leading to the development of causal illusions could be responsible for the endorsement of pseudoscientific beliefs. Nevertheless, it could also be the case that some individuals tend to develop causal illusions in our task because they hold prior pseudoscientific beliefs. Moreover, both causal illusion and pseudoscientific beliefs could stem from a different mechanism not contemplated in our research.

All in all, our results show a reliable association between pseudoscientific beliefs and causal illusion. In our view, this observation could indicate that believers in pseudoscience might present a bias in the interpretation of a given piece of contingency information, leading to stronger perception of a causal relation between non-contingent events, at least when the task is 
framed in pseudoscientific terms. We extend previous observations regarding individuals who believe in the paranormal to a set of unwarranted beliefs which appear to be more relevant and present in our daily lives. 


\section{References}

Aarnio, K., \& Lindeman, M. (2005). Paranormal beliefs, education, and thinking styles. Personality and Individual Differences, 39(7), 1227-1236. https://doi.org/10.1016/J.PAID.2005.04.009

Allan, L. (1993). Human contingency judgments: Rule based or associative? Psychological Bulletin, 114, 435-448.

Barberia, I., Tubau, E., Matute, H., \& Rodríguez-Ferreiro, J. (2018). A short educational intervention diminishes causal illusions and specific paranormal beliefs in undergraduates. PLoS ONE, 13(1), e0191907. https://doi.org/10.1371/journal.pone.0191907

Barberia, I., Vadillo, M., \& Rodríguez-Ferreiro, J. (2019). Persistence of Causal Illusions After Extensive Training. Frontiers in Psychology, 10, 24. https://doi.org/10.3389/fpsyg.2019.00024

Blackmore, S., \& Trościanko, T. (1985). Belief in the paranormal: Probability judgements, illusory control, and the 'chance baseline shift.' British Journal of Psychology, 76(4), 459468. https://doi.org/10.1111/j.2044-8295.1985.tb01969.x

Blanco, F., Barberia, I., \& Matute, H. (2015). Individuals who believe in the paranormal expose themselves to biased information and develop more causal illusions than nonbelievers in the laboratory. PloS One, 10(7), e0131378. https://doi.org/10.1371/journal.pone.0131378

Blanco, F., Matute, H., \& Vadillo, M. A. (2013). Interactive effects of the probability of the cue and the probability of the outcome on the overestimation of null contingency. Learning and Behavior, 41, 333-340. https://doi.org/10.3758/s13420-013-0108-8.

Bressan, P. (2002). The connection between random sequences, everyday coincidences, and belief in the paranormal. Applied Cognitive Psychology, 16(1), 17-34. https://doi.org/10.1002/acp.754

Brugger, P., \& Graves, R. E. (1997). Testing vs. believing hypotheses: magical ideation in the judgement of contingencies. Cognitive Neuropsychiatry, 2, 251-272. https://doi.org/10.1080/135468097396270

Brugger, P., Landis, T., \& Regard, M. (1990). A 'sheep-goat effect' in repetition avoidance: Extra-sensory perception as an effect of subjective probability? British Journal of Psychology, 81(4), 455-468. https://doi.org/10.1111/j.2044-8295.1990.tb02372.x

Brugger, P., Regard, M., Landis, T., Cook, N., Krebs, D., \& Niederberger, J. (1993). 'Meaningful' patterns in visual noise: Effects of lateral stimulation and the observer's belief in ESP. Psychopathology, 26, 261-265. https://doi.org/10.1159/000284831

Díaz-Vilela, L., \& Álvarez, C. J. (2004). Differences in paranormal beliefs across fields of study from a Spanish adaptation of Tobacyk's RPBS. The Journal of Parapsychology, 68(1), 405-421.

Fasce, A., \& Picó, A. (2019). Conceptual foundations and validation of the Pseudoscientific Belief Scale. Applied Cognitive Psychology. https://doi.org/10.1002/acp.3501

FECYT. (2017). Percepción social de la ciencia y la tecnología - 2016.

Ferrero, M., Garaizar, P., \& Vadillo, M. A. (2016). Neuromyths in Education: Prevalence among Spanish Teachers and an Exploration of Cross-Cultural Variation. Frontiers in Human Neuroscience, 10, 496. https://doi.org/10.3389/fnhum.2016.00496

Griffiths, O., Shehabi, N., Murphy, R. A., \& Le Pelley, M. E. (2018). Superstition predicts 
perception of illusory control. British Journal of Psychology, 110(3), 499-518. https://doi.org/10.1111/bjop.12344

Lilienfeld, S., Lynn, S., Ruscio, J., \& Beyerstein, B. (2010). 50 Great Myths of Popular Psychology. Hoboken, NJ: Wiley-Blackwell. https://doi.org/10.1080/10888700902955989

Lindeman, M. (2011). Biases in intuitive reasoning and belief in complementary and alternative medicine. Psychology \& Health, 26(3), 371-382. https://doi.org/10.1080/08870440903440707

Majima, Y. (2015). Belief in Pseudoscience, Cognitive Style and Science Literacy. Applied Cognitive Psychology, 29(4), 552-559. https://doi.org/10.1002/acp.3136

Matute, H., Blanco, F., Yarritu, I., Díaz-Lago, M., Vadillo, M. A., \& Barberia, I. (2015). Illusions of causality: How they bias our everyday thinking and how they could be reduced. Frontiers in Psychology, 6(888). https://doi.org/10.3389/fpsyg.2015.00888

Matute, H., Yarritu, I., \& Vadillo, M. A. (2011). Illusions of causality at the heart of pseudoscience. British Journal of Psychology, 102, 392-405. https://doi.org/10.1348/000712610X532210

NCCIH-NIH. (2008). The use of complementary and alternative medicine in the United States. Retrieved from https://nccih.nih.gov/research/statistics/2007/camsurvey_fs1.htm

Posadzki, P., Alotaibi, A., \& Ernst, E. (2012). Prevalence of use of complementary and alternative medicine (CAM) by physicians in the UK: a systematic review of surveys. Clinical Medicine (London, England), 12(6), 505-512. https://doi.org/10.7861/clinmedicine.12-6-505

Sierro, G., Rossier, J., Mason, O. J., \& Mohr, C. (2016). French validation of the O-LIFE short questionnaire. European Journal of Psychological Assessment, 32(3), 195-203. https://doi.org/10.1027/1015-5759/a000249

Tobacyk, J. (2004). A Revised Paranormal Belief Scale. International Journal of Transpersonal Studies, 23, 94-98. https://doi.org/10.24972/ijts.2004.23.1.94

van Prooijen, J.-W., Douglas, K. M., \& De Inocencio, C. (2018). Connecting the dots: Illusory pattern perception predicts belief in conspiracies and the supernatural. European Journal of Social Psychology, 48(3), 320-335. https://doi.org/10.1002/ejsp.2331

Wagenmakers, E.-J., Love, J., Marsman, M., Jamil, T., Ly, A., Verhagen, J., ... Morey, R. D. (2018). Bayesian inference for psychology. Part II: Example applications with JASP. Psychonomic Bulletin \& Review, 25(1), 58-76. https://doi.org/10.3758/s13423-017-1323-7

Wiseman, R., \& Watt, C. (2006). Belief in psychic ability and the misattribution hypothesis: A qualitative review. British Journal of Psychology, 97(3), 323-338. https://doi.org/10.1348/000712605X72523 
Figure 1. Association between the intensity of the causal illusion and the scores in the PES and SBQ questionnaires.
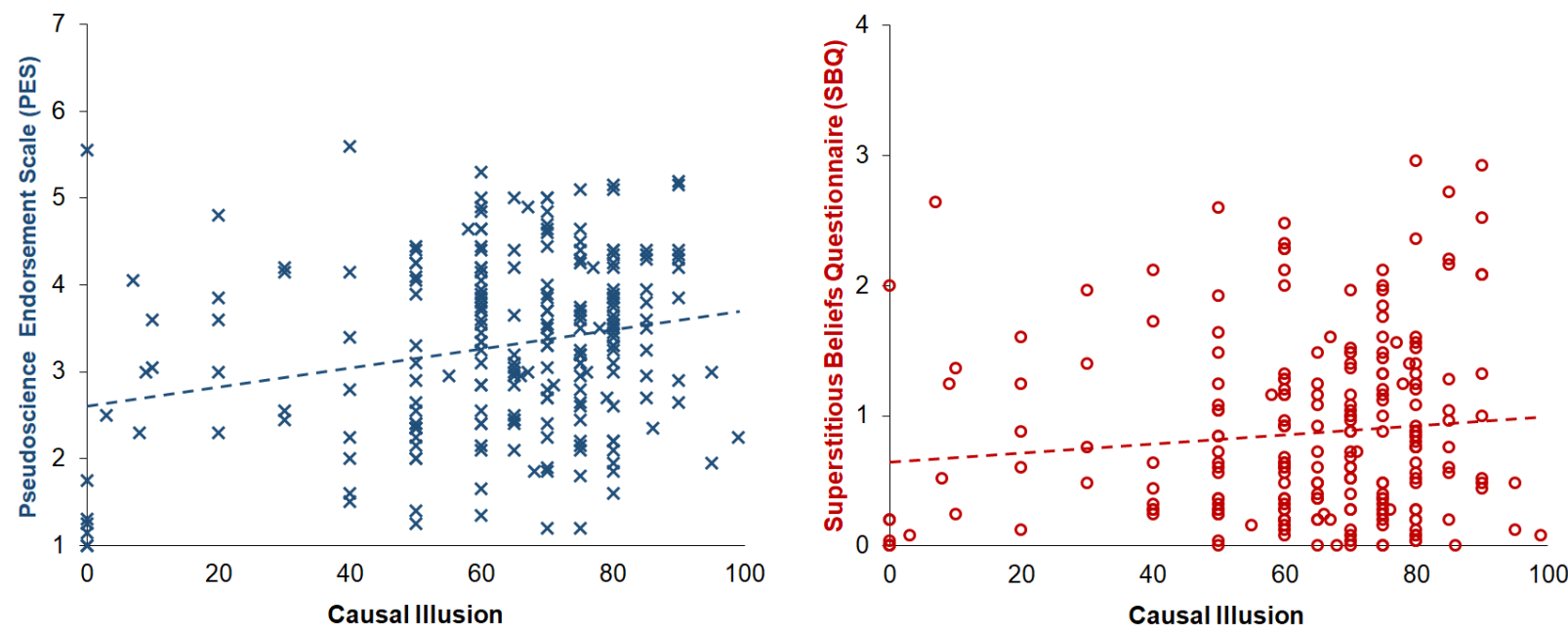